\title{
Hearing outcomes between multi-session and single-session radiosurgery for vestibular schwannoma: a single center study
}

\author{
Xuqun Tang ${ }^{1 \#}$, Mingzhe Zheng ${ }^{2 \#}$, Hailiang Tang ${ }^{2 \#}$, Hanfeng $\mathrm{Wu}^{1}$, Nan Zhang $^{1,2}$, Jiazhong Dai $^{1,2}$, Li Pan $^{1,2}$ \\ ${ }^{1}$ Department of Neurosurgery, Shanghai Gamma Hospital, Gamma Knife Centre of Huashan Hospital, Shanghai 200040, China; ${ }^{2}$ Department of \\ Neurosurgery, Huashan Hospital of Fudan University, Shanghai 200040, China \\ Contributions: (I) Conception and design: X Tang; (II) Administrative support: J Dai, L Pan; (III) Provision of study materials or patients: H Wu, N \\ Zhang; (IV) Collection and assembly of data: M Zheng; (V) Data analysis and interpretation: H Tang; (VI) Manuscript writing: All authors; (VII) \\ Final approval of manuscript: All authors. \\ "These authors contributed equally to this work. \\ Correspondence to: Li Pan. Department of Neurosurgery, Huashan Hospital of Fudan University, No.12 Middle Wulumuqi Road, Shanghai 200040, \\ China. Email: lipanmr@sina.com.
}

Background: Hearing preservation rate after Gamma Knife radiosurgery (GKRS) for vestibular schwannoma (VS) remains low. The aim of this study was to study hearing outcomes between multisession and single session GKRS in patients with VS and determine prognostic factors associated with hearing preservation.

Methods: A retrospective analysis of 561 consecutive patients with VS subjected to multisession (74 patients) or single session (487 patients) GKRS (from June 2011 to April 2015) was performed. Propensity score matching (PSM) based on patient age, gender, tumor volume and pre-GKRS hearing was used to compare the two groups. The tumor control rate and complications were evaluated, especially hearing preservation and its prognostic factors.

Results: According to PSM, 29 patients from each group with similar characteristics were selected for the study. Patients' age, tumor volume, pre-GKRS pure tone average (PTA) and radiographic follow-up period were not significantly different between the two groups $(\mathrm{P}>0.05)$. The tumor control rate was $75.9 \%$ for the multisession group compared with $62.1 \%$ for the single session group $(\mathrm{P}=0.1142) ; 20$ patients had a less than $20 \mathrm{~dB}$ change in PTA in multisession group, with the hearing preservation rate of $69.0 \%$ (20/29) compared with $65.5 \%(19 / 29)$ in single session group $(\mathrm{P}=0.08)$. Multivariate analysis revealed that linear internal auditory canal (IAC) length was the only significant predictor of hearing loss in the multisession group. At last follow-up, one patient complaining of sudden hearing loss was diagnosed with tumor bleeding 12 months after multisession GKRS. One patient from multisession group and two patients from single session group suffered from temporal facial numbness. Facial spasm developed in one patient after multisession GKRS.

Conclusions: Our results supported that multisession GKRS could be an effective and safe treatment option for VS compared to single session GKRS. The hearing preservation rate after multisession GKRS was not superior to single session radiosurgery in our short-term follow-up study. However, patients with longer IAC length may benefit from multisession strategy in terms of hearing preservation.

Keywords: Vestibular schwannoma (VS); gamma knife; hearing preservation; multisession gamma knife radiosurgery (multisession GKRS)

Submitted Jun 11, 2018. Accepted for publication Aug 07, 2018.

doi: $10.21037 /$ tcr.2018.08.15

View this article at: http://dx.doi.org/10.21037/tcr.2018.08.15 


\section{Introduction}

Since its advent in 1969, Gamma Knife radiosurgery (GKRS) has become a safe and effective treatment modality for patients with vestibular schwannoma (VS) compared to open microsurgical resection, showing excellent long-term tumor control rates $(93-97 \%$ at 10 years) $(1,2)$. Due to the development of modern planning techniques (e.g., lower marginal dose) and high-resolution imaging, cranial nerve morbidity has been significantly decreased while retaining high rate of tumor control (3-6). However, overall hearing preservation rates have been more complicated (32-83\%), and much lower than other cranial nerves after GKRS for VS (3,7-14).

Several risk factors have been implicated for postGKRS hearing loss, including patient age, tumor volume, pretreatment hearing status, post-treatment transient volume expansion (TVE), radiation dose, direct radiationinduced acoustic nerve damage, radiation-induced changes in the cochlear vascular supply, direct radiation-induced cochlear damage, and irradiation of brainstem auditory nuclei (15-20).

Recently, multisession radiosurgery has been explored in an attempt to achieve tumor control rate equal to single session radiosurgery while decreased the risk of cranial nerves damage $(21,22)$. Gamma knife has been used exclusively for single session radiosurgery, and there is rare report of multisession GKRS for patient with VS. In this study, we reported the hearing outcomes of VS patients treated with multisession GKRS compared with single session GKRS, and investigated specific factors that could influence hearing preservation rate.

\section{Methods}

\section{Patient characteristics}

From June 2011 to April 2015, 561 VS patients were subjected to multisession (74 patients) or single session (487 patients) GKRS at Shanghai Gamma Hospital. To balance the difference of the basic data between two groups, which may impact the results, propensity score matching (PSM) was utilized to reduce selection bias between the two groups, which was based on patient age, gender, tumor volume, pre-GKRS hearing and length of follow-up. After PSM, there are 27 patients $(93.1 \%)$ with GardnerRobertson (GR) class I or II hearing (serviceable hearing) before GKRS in the multisession group and $20(69.0 \%)$ in the single session group. The $\mathrm{P}$ value is 0.0190 between the two groups. Due to the small number [58] of patients in final data analysis, we considered $\mathrm{P}$ value smaller than 0.01 as of significant difference when we use PSM. The final study population included 29 patients from each group with similar demographic characteristics, followed up with both audiogram and MRI scans in our hospital. Patient characteristics in each group are displayed in Table 1. The diagnoses were established mainly based on MRI findings and clinical presentation except for one patient in single session group who undergone previous microsurgical resection.

All patients were subjected to clinical examination and MRI scans before and after GKRS. Audiographic results were evaluated according to the pure tone average (PTA) as well as the GR classification. PTA was calculated by averaging the threshold hearing score in decibels at 500, 1,000, and 2,000 Hz. Hearing outcomes were divided into two different ways: difference in pre- and post-treatment PTA and GR classification. If the difference between the pre-treatment and post-treatment PTA was less than $20 \mathrm{~dB}$, the patient was categorized as hearing preserved $(12,15,23)$.

All the patients included in our study are consent informed, and the study was conducted under the supervision of Ethics Committee of Huashan Hospital, Fudan University.

\section{Gamma knife treatments}

Treatments were performed under local anesthesia, using the Leksell Gamma Knife model C (Elekta AB, before April 2012) or Perfexion (from May 2012) at Shanghai Gamma Hospital. Before treatment, high resolution gadoliniumenhanced T1-weighted and T2-weighted MRI images were obtained for each patient on 1.5 tesla MRI imaging system (Signa Excite, GE, USA). Constructive inference in steady state (CISS) sequences were also obtained to delineate cranial nerves. Cochlear volumes were drawn and the linear lengths of internal auditory canal (IAC) were measured using the T2-weighted MRI images (Figure 1). Dose planning was performed using the Leksell GammaPlan software (Version 10.0, Elekta Instruments AB, Stockholm, Sweden).

In multisession group, radiation was delivered in 3 sessions over 3 consecutive days. The dose planning strategy was similar to single session radiosurgery except for the prescription dose per session, which was decided based on the biologically equivalent dose as a singlefraction treatment for tissues with an $\alpha / \beta$ ratio of $3 \mathrm{~Gy}$. The mean marginal dose per session was 6.7 Gy (range, 6.3 to 
Table 1 Patient characteristics/Gamma knife parameters

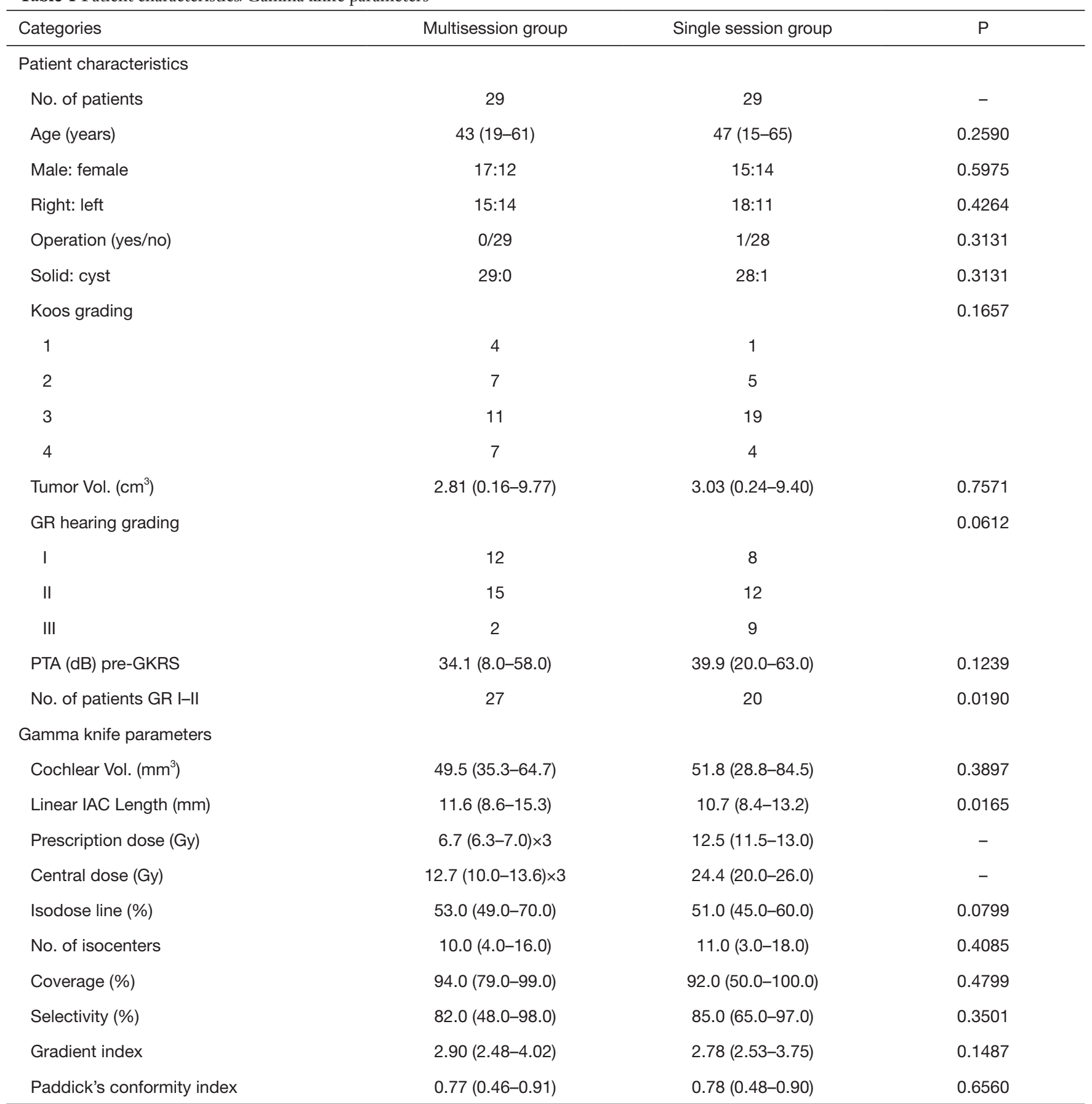

GR, Gardner-Robertson; PTA, pure tone average; GKRS, Gamma Knife radiosurgery; IAC, internal auditory canal

7 Gy) directed to the $49.0-70.0 \%$ isodose line (mean $53 \%$ ), with an average tumor volume of $2.81 \mathrm{~cm}^{3}$ (range, 0.16 to $\left.9.77 \mathrm{~cm}^{3}\right)$. Dexamethasone $(2.5 \mathrm{mg}$ per day) was administered during the whole sessions. Inter-fractional displacement errors were found to be less than $0.5 \mathrm{~mm}$. In single session group, the mean marginal dose was 12.5 Gy (range, 11.5 to $13 \mathrm{~Gy}$ ) directed to the $45.0-60.0 \%$ isodose line (mean $51 \%$ ), with an average tumor volume of $3.03 \mathrm{~cm}^{3}$ (range, 0.24 to $9.40 \mathrm{~cm}^{3}$ ).

In multisession group, dose-volume histograms were 

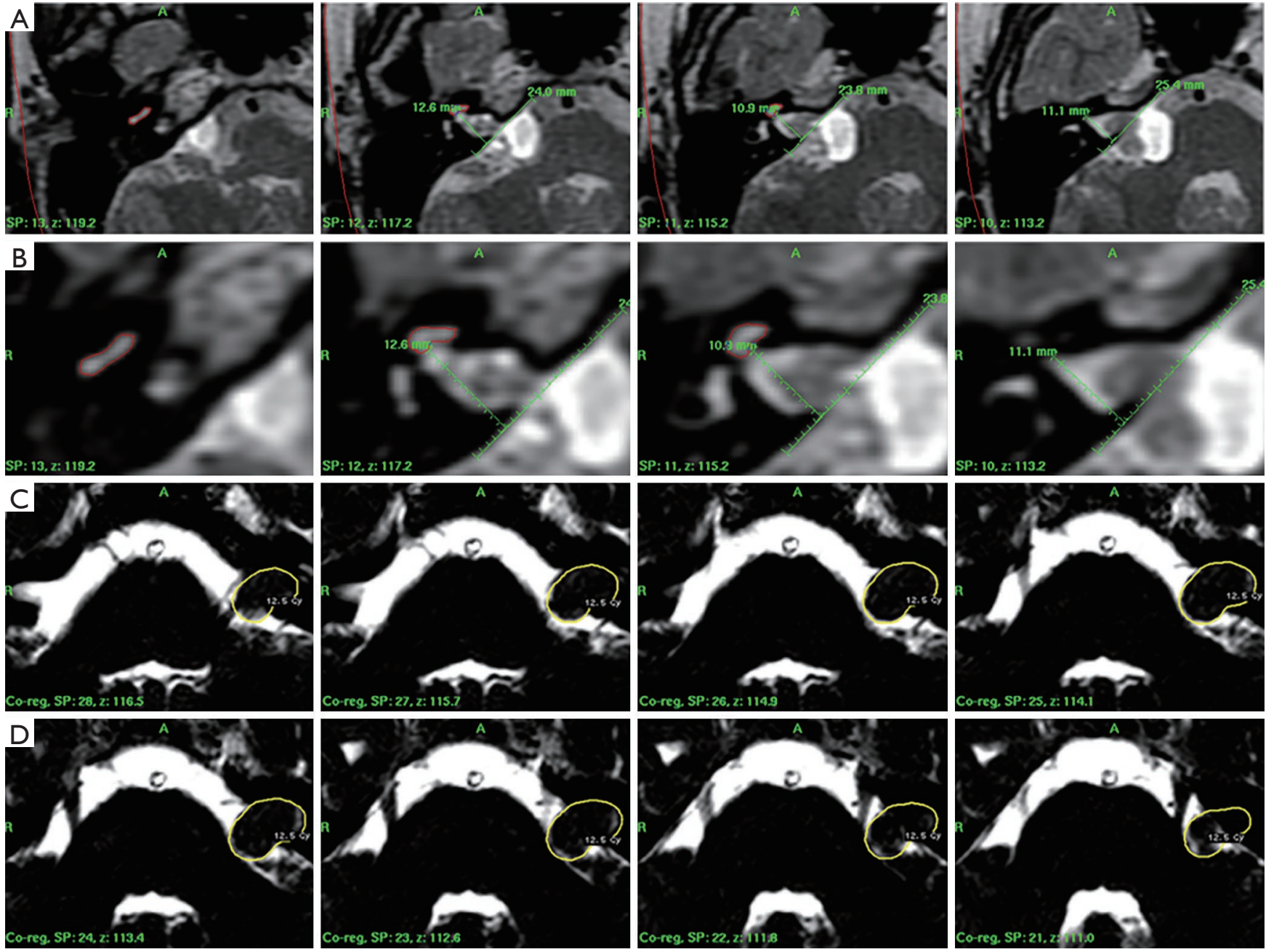

Figure 1 Screen image captured from GammaPlan version 10.0 demonstrating the method of drawing the cochlea and measuring the linear length of IAC using T2-weighted magnetic resonance images (A,B) and the intersection of the isodose line with cranial nerves using magnetic resonance CISS sequence. IAC, internal auditory canal; CISS, constructive inference in steady state.

created for the cochlea using T2-weighted MRI images and the Leksell GammaPlan software. Cochlear volume receiving 1.5 and 2.0 Gy per session or greater were noted. These cutoff points were chosen based on other reports $(10,15)$. The maximum, minimum and mean radiation dose to the cochlea were also recorded. Plan conformality was determined by four measures: Paddick's conformity index (PCI), coverage, selectivity and gradient index (GI). The Gamma Knife parameters were displayed in Table 1.

\section{Follow up}

Periodic clinical, audiographic and radiological follow-up were carried out for each patient at 6-month intervals for 2 years then annually thereafter. The PTA and GR class were recorded, and the endpoints of $20 \mathrm{~dB}$ or less loss in PTA, preserved GR hearing class were used. TVE and postGKRS peritumor edema were also recorded. To determine prognostic factors for hearing preservation in multisession group, univariate and multivariate analysis were performed. Tumor progression was determined using gadoliniumenhanced MRI and defined as an increase in tumor volume presented at the time of last follow-up. Facial nerve function was assessed according to the House-Brackmann (HB) grading system. Trigeminal nerve function was assessed by patient examination and reports. 


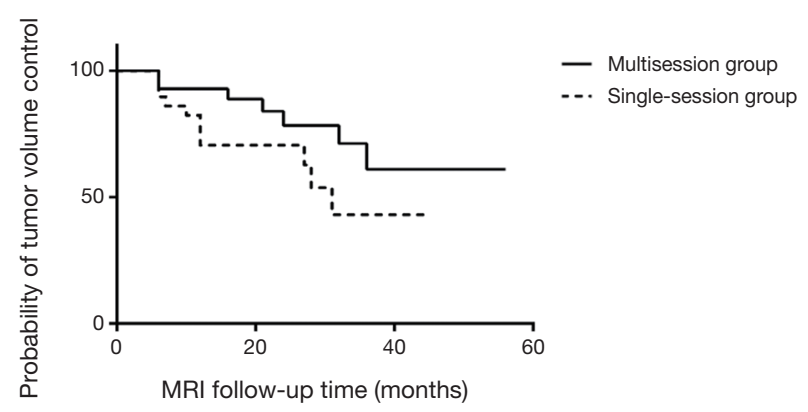

Figure 2 Graph depicting Kaplan-Meier curves of cumulative radiographic tumor controls in 29 patients after multisession or single session GKRS for VS. Log-rank test: $\mathrm{P}=0.1142$. GKRS, Gamma Knife radiosurgery; VS, vestibular schwannoma.

\section{Statistical analysis}

The Kaplan-Meier method was used to calculate and compare estimated rates of radiographic tumor control and hearing preservation between multisession and single session groups. In the multisession group, the simple comparison of continuous variables between the hearing preserved and not preserved groups ( $\geq 20 \mathrm{~dB}$ loss) was performed using two tailed $t$-test, whereas in case of frequencies or proportions for categorical data, Fisher's exact test was used. Based on the $\mathrm{P}$ values, the 5 continuous variables with $\mathrm{P}<0.1$ were included in the multivariate logistic regression (Stata, logistic). The dependent variable was hearing loss as defined as patients with $\geq 20 \mathrm{~dB}$ loss. Finally, only one of the 5 variables was considered significantly different between the hearing preserved and not preserved groups ( $\geq 20 \mathrm{~dB}$ loss) after multisession GKRS. We used statistical software package Stata 10 (StataCorp, College Station, Texas) and Prism 4 for Windows (GraphPad software, Inc, USA) for the analysis.

\section{Results}

\section{Radiographic tumor control}

The mean duration of MRI scan follow-up was 26 months (range, 5 to 56 months) in the multisession group and 19 months (range, 6 to 45 months) in the single session group $(\mathrm{P}=0.0657)$. At the latest follow-up, tumor volumes of 22 patients in multisession group and 18 patients in single session group were decreased. The total radiographic tumor control rate was $75.9 \%(22 / 29)$ in multisession group and $62.1 \%(18 / 29)$ in single session group. The Kaplan-Meier method showed the difference was not significant between the two groups, but in multisession group there was a trend toward improved radiographic tumor control rates $(75.9 \%$ in multisession group vs. $62.1 \%$ in single session group; $\mathrm{P}=0.1142$ ) (Figure 2).

We observed post-treatment TVE in 12 patients (41.4\%) from the multisession group and 11 patients (37.9\%) from the single session group $(\mathrm{P}=0.7884)$. Five cases of postGKRS peritumoral edema in the multisession group and four cases in the single session group were identified $(\mathrm{P}=0.7169)$. However, none of these patients required additional surgical treatment after GKRS except for shortterm course of mannitol and dexamethasone.

One patient in multisession group suffered from sudden hearing loss 12 months after GKRS. Follow-up MRI images revealed intratumoral hemorrhage and continuous tumor enlargement. Microsurgical resection was recommended for the patient. Thus, tumor control rate in terms of requiring further treatment was $96.6 \%$ in the multisession group, while $100 \%$ tumor control rate was achieved in the single session group.

\section{Comparison of GR class I or II bearing preservation outcomes}

The mean follow-up period for audiometry was 21 months (range, 5 to 49 months) in the multisession group and 16 months (range, 6 to 33 months) in the single session group $(\mathrm{P}=0.0385)$. In the multisession group, 27 patients had GR class I or II hearing (serviceable hearing) before GKRS, and 14 patients had GR class I or II hearing after GKRS during the follow-up period. The hearing preservation rate for these patients was $51.9 \%$ (Table 2). In the single session group, 20 patients had GR class I or II hearing before GKRS, and 9 patients had GR class I or II hearing after GKRS at last follow-up, with hearing preservation rate of $45.0 \%$ (Table 2). According to the Kaplan-Meier survival plots, the difference of serviceable hearing preservation rate was not significant between the two groups ( $\mathrm{P}=0.1015)$ (Figure 3).

\section{Comparison of $<20 \mathrm{~dB}$ change in PTA hearing preservation outcomes}

Besides the GR classification scale, we also analyzed hearing outcomes using PTA. Based on previous studies $(13,22,23)$, a less than $20 \mathrm{~dB}$ difference in PTA between pre-treatment and post-treatment audiograms was defined as having hearing preservation, whereas a change in PTA of $20 \mathrm{~dB}$ or greater was considered as no preservation. In the 
Table 2 Results for hearing preservation/tumor control/complications

\begin{tabular}{|c|c|c|c|}
\hline Categories & Multisession group & Single session group & $\mathrm{P}$ \\
\hline Audiogram follow-up (months) [range] & $21[5-49]$ & 16 [6-33] & 0.0385 \\
\hline PTA (dB) post-GKRS & $48.0[13.0-78.0]$ & $55.1[180-90.0]$ & 0.1621 \\
\hline Change in PTA & $13.9[-20.0$ to 57.0$]$ & $15.2[-7.0$ to 64.0$]$ & 0.7564 \\
\hline Preservation rate of GR I-II post-GKRS & $51.9 \%(14 / 27)$ & $45.0 \%(9 / 20)$ & 0.1015 \\
\hline \multicolumn{4}{|l|}{ Radiographic outcomes } \\
\hline MRI follow-up time (months) & $26[5-56]$ & $19[6-45]$ & 0.0657 \\
\hline Tumor Vol. $\left(\mathrm{cm}^{3}\right)$ post-GKRS & $2.33[0.16-13.12]$ & $2.55[0.15-9.74]$ & 0.7609 \\
\hline \multicolumn{4}{|l|}{ Complications } \\
\hline No. of peritumor edema & $5(17.2)$ & $4(13.8)$ & - \\
\hline \multicolumn{4}{|l|}{ Facial nerve complication } \\
\hline Temporary & 0 & 0 & - \\
\hline Permanent & 1 & 0 & - \\
\hline \multicolumn{4}{|l|}{ Trigeminal nerve complication } \\
\hline Temporary & 1 & 2 & - \\
\hline Permanent & - & - & - \\
\hline
\end{tabular}

Data are presented as mean [range] or $\mathrm{n}(\%)$. GR, Gardner-Robertson; PTA, pure tone average; GKRS, Gamma Knife radiosurgery; TVE, transient tumor volume expansion.

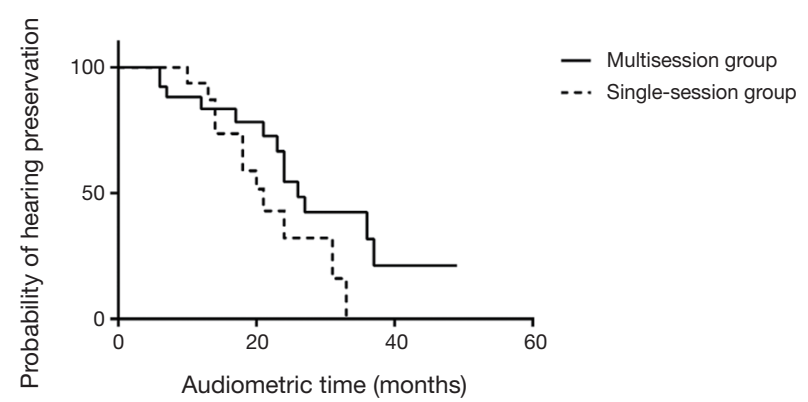

Figure 3 Graph showing Kaplan-Meier curves of cumulative GR class I or II hearing preservation in 27 patients who underwent multisession GKRS and 20 patients who underwent single session GKRS for VS. Log-rank test: P=0.1015. GKRS, Gamma Knife radiosurgery; VS, vestibular schwannoma; GR, GardnerRobertson. multisession group, 20 patients had a less than $20 \mathrm{~dB}$ change in the PTA. Overall hearing preservation rate for these patients with any measurable hearing and a less than $20 \mathrm{~dB}$ loss change in their PTA was $69.0 \%$ (20/29). In the single session group, 19 patients had a less than change in their PTA, with an overall hearing preservation rate of $65.5 \%$ (19/29). However, no statistically significant difference in hearing preservation rate was found between the two groups $(\mathrm{P}=0.08)$ (Figure 4).

\section{Prognostic factors of hearing preservation in the multisession group}

We analyzed and compared 25 variables between the 


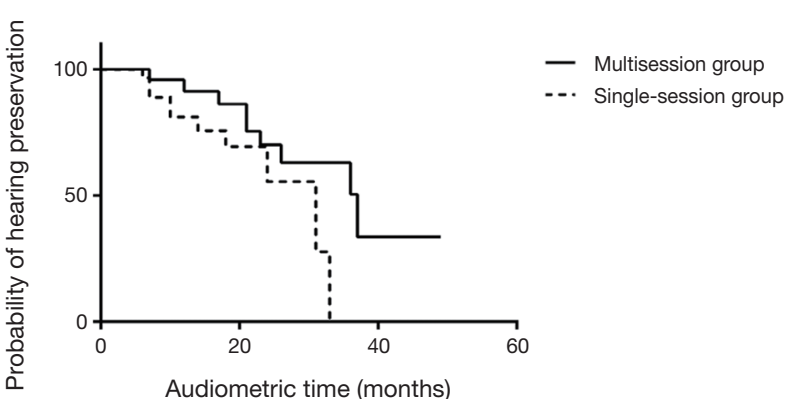

Figure 4 Graph showing Kaplan-Meier curves of cumulative $<20 \mathrm{~dB}$ change in PTA hearing preservation in 29 patients after multisession or single session GKRS for VS. Log-rank test: $\mathrm{P}=0.08$. GKRS, Gamma Knife radiosurgery; VS, vestibular schwannoma; PTA, pure tone average.

deteriorated ( $\geq 20 \mathrm{~dB}$ loss in PTA) and the preserved hearing groups ( $<20 \mathrm{~dB}$ loss in PTA) after multisession GKRS using the two tailed $t$-test for continuous variables and Fisher's exact test for categorical data (Table 3). Five variables were noted on univariate analysis towards statistical significance between the two groups $(\mathrm{P}<0.1)$ : tumor volume, linear IAC length, no. of isocenters, selectivity and PCI. Other variables failed to demonstrate significance as prognostic factors. The five variables were then chosen for entry into a multivariate logistic regression model. Results showed that linear IAC length was the only significant predictor for hearing loss after multisession GKRS in this model, with an odds ratio of 0.3868 (Table 3).

\section{Trigeminal nerve and facial nerve preservation rates}

A temporary trigeminal nerve complication rate of $3.4 \%$ ( 1 of 29 patients) in the multisession group and $6.9 \%$ ( 2 of 29 patients) in the single session group were observed (Table 2). All the three patients had resolution of their facial numbness at last follow-up. Facial spasm, which was still present at the time of last follow-up, developed in one patient after multisession GKRS (Table 2). Neither transient nor permanent facial palsy developed in patients from either group. Thus, the permanent facial nerve complication rate was $3.4 \%$ ( 1 of 29 patients) in the multisession group.

\section{Discussions}

Recent trials of multisession radiosurgery for perioptic lesions have been conducted in an attempt to achieve tumor control rates equal to single session radiosurgery while maintaining the risk of optic neuropathy as low as in fractionated radiotherapy $(21,22)$. GKRS for VS has been used exclusively for single session radiosurgery because of the inconvenience of stereotactic frame fixation. There has been no report of multisession GKRS for the purpose of preserving hearing in patients with VS. In our study, we reported in detail the hearing outcome of 29 VS patients treated with multisession GKRS compared with single session GKRS. The results would be informative and instructive in the upcoming era of fractionated GKRS.

\section{Hearing preservation after GKRS for VS}

It has been more than 40 years since the first GKRS for VS was reported in 1969. Since then, the number of VS patients treated with GKRS has increased over the past decades due to its low morbidity and high tumor control rate. In modern series of GKRS for VS, facial and trigeminal nerve preservation rates have been reported to range from $95 \%$ to $100 \%$, and rates of hearing loss have also declined $(5,8,12,15)$. Advances in stereotactic imaging, targeting, and dose-planning software provide means of lowering the marginal dose for VS, which could be the explanation (3-6,15). However, the hearing preservation rate still lower than other cranial nerve modalities $(8,15,24)$. Brown et al. (15) proposed a practical hearing preservation criterion: patient is categorized as hearing preserved if the difference in PTA between the pre-treatment and post-treatment audiograms was less than $20 \mathrm{~dB}(<20 \mathrm{~dB})$. This " $<20 \mathrm{~dB}$ change in PTA" criteria is favored because it yields a continuous outcome variable and allows the detection of subtle changes in hearing thresholds before and after GKRS, especially when the follow-up time is relatively short. The use of the change in PTA as the outcome variable also allows inclusion of patients who still had testable hearing in the treated ear but were not categorized as GR class I or II.

In our study, we analyzed and compared the hearing preservation rates between multisession and single session GKRS using both " $<20 \mathrm{~dB}$ change in PTA" and "GR class I or II" criteria of hearing preservation. The hearing preservation rate results of our study are comparable to those of previous reports. Based on either criteria, we failed to show a significant difference in hearing preservation rates between the two groups (PTA: $69.0 \%$ vs. $65.5 \%, \mathrm{P}=0.08$; GR class: $51.9 \%$ vs. $45 \%, \mathrm{P}=0.1015)$. However, there was a trend toward improved hearing preservation rates in multisession group (Figure 4). The audiometric follow-up time in multisession group was significantly longer than 
Table 3 Univariate/multivariate comparison between $<20 \mathrm{~dB}$ and $\geq 20 \mathrm{~dB}$ PTA groups after multisession GKRS

\begin{tabular}{|c|c|c|c|c|c|c|}
\hline \multirow[t]{2}{*}{ Categories } & \multirow{2}{*}{ All patients } & \multirow{2}{*}{$\begin{array}{l}\text { Hearing preserved } \\
\text { (<20 dB PTA })\end{array}$} & \multirow{2}{*}{$\begin{array}{l}\text { Hearing loss } \\
(\geq 20 \mathrm{~dB} \text { PTA) }\end{array}$} & \multirow{2}{*}{$\mathrm{P}$} & \multicolumn{2}{|c|}{$\begin{array}{l}\text { Multivariate logistic } \\
\text { regression }\end{array}$} \\
\hline & & & & & Odds ratio & $\mathrm{P}$ \\
\hline Koos grading (1:2:3:4) & $4: 7: 11: 7$ & $4: 6: 6: 4$ & $0: 1: 5: 3$ & 0.2364 & - & - \\
\hline TVE (yes:no) & $12: 17$ & $9: 11$ & $3: 6$ & 0.5551 & - & - \\
\hline Peritumor edema (yes:no) & $5: 24$ & $3: 17$ & $2: 7$ & 0.6338 & - & - \\
\hline Age (years) & 43.4 & 43.0 & 44.3 & 0.7857 & - & - \\
\hline Linear IAC length (mm) & 11.63 & 11.98 & 10.87 & 0.0726 & 0.3868 & 0.090 \\
\hline Prescription dose (Gy) & 6.71 & 6.72 & 6.70 & 0.7627 & - & - \\
\hline Central dose (Gy) & 12.71 & 12.74 & 12.63 & 0.7801 & - & - \\
\hline Isodose line (\%) & 53.14 & 53.10 & 53.22 & 0.9498 & - & - \\
\hline No. of isocenters & 10 & 9 & 12 & 0.0980 & - & 0.869 \\
\hline Coverage & 0.9383 & 0.9315 & 0.9533 & 0.2937 & - & - \\
\hline Selectivity & 0.8231 & 0.7980 & 0.8789 & 0.0977 & - & 0.679 \\
\hline $\begin{array}{l}\text { Mean percentage of volume of cochlear } \\
\text { receiving }>1.5 \text { Gy }\end{array}$ & $76 \%$ & $74 \%$ & $82 \%$ & 0.4681 & - & - \\
\hline $\begin{array}{l}\text { Mean percentage of volume of cochlear } \\
\text { receiving }>2.0 \mathrm{~Gy}\end{array}$ & $38 \%$ & $37 \%$ & $40 \%$ & 0.8399 & - & - \\
\hline Mean integral dose of tumor (Gy) & 9.14 & 9.16 & 9.10 & 0.8222 & - & - \\
\hline
\end{tabular}

In the univariate analysis, two-tailed $t$-tests and Fisher's exact tests were performed in the two groups. For the multivariate analysis, only the variables with $\mathrm{P}$ value $<0.1$ shown in italics were entered into the logistic regression model. Linear IAC length was the only significant predictor of hearing loss in this model, with an odds ratio of 0.3868. GR, Gardner-Robertson; PTA, pure tone average; GKRS, Gamma Knife radiosurgery; TVE, transient tumor volume expansion; IAC, internal auditory canal.

that in single session group ( 21 vs. 16 months, $\mathrm{P}=0.0385$ ). Hence, future prospective and comparative studies with more cases and longer follow-up periods (especially in single session group) are needed to address any possible benefit of multisession GKRS strategy on hearing.

\section{Prognostic factors of hearing preservation after multisession GKRS}

Previous studies have suggested possible prognostic factors of hearing preservation, such as the GR grade, radiation 
dose to the cochlea, transient TVE after GKRS, length of irradiated cochlear nerve, marginal dose to the tumor, and age. However, there is still no clear mechanism for patients losing their hearing after GKRS for VS. Han et al. (8) hypothesized that patients with considerable intracanalicular pressure at the time of GKRS are prone to lose their serviceable hearing, due to the added intracanalicular pressure induced by TVE, which usually occurs within the first 12 months after GKRS for VS. A predictive model for hearing preservation after GKRS for VS is needed to guide appropriate patient selection, timing and dose planning of GKRS, which will lead to better of hearing preservation in patients with VS.

In our study, the rate of TVE after multisession GKRS was comparable to that in the single session group and TVE was not a prognostic factor of hearing preservation after multisession GKRS. However, this result should be interpreted in combination with auditory brainstem response (ABR) data and longer follow-up times.

Twenty-five variables potentially related to hearing preservation after GKRS were analyzed and compared between hearing-preserved and hearing-lost groups after multisession GKRS. Multivariate analysis identified shorter linear IAC length as the only significant predictor of hearing loss after multisession GKRS, which implies that patients with longer IAC length may benefit from this new strategy in terms of hearing preservation.

\section{Multisession GKRS for VS Patients Who Retain Serviceable Hearing}

In our series, multisession GKRS over 3 days was tolerated well by all patients. In addition to safety and efficacy in the management of VS, multisession GKRS shortened the treatment period from 5 to 6 weeks by conventional fractionated radiotherapy to 3 days. Linskey et al. argued that it was the prescription dose conformality with precise delivery rather than fractionation that allowed for hearing preservation in VS stereotactic radiosurgery (25). Compared to other modalities, gamma knife treatment has the advantages of minimal interfractional displacement error and higher precision of radiation delivery to the target. Multisession GKRS integrates the advantages of conventional fractionated radiotherapy and single session GKRS that favors hearing preservation for VS patients undergoing radiosurgery. In this study, we evaluated hearing preservation and tumor control after multisession GKRS during the short-term follow-up period, and the outcome was favorable in most patients. Only one patient failed with intratumoral hemorrhage and continuous tumor enlargement. However, we still observed favorable trend towards higher hearing preservation rate in the multisession group.

Although our short-term follow-up study suggested that multisession GKRS yields hearing preservation and tumor control rates equivalent to single dose GKRS, significant questions remain regarding long-term tumor control and complications $(25,26)$. In addition, this preliminary study is limited by its retrospective, case-control design and its findings need to be verified in the prospective fashion. The final data analysis of relatively small proportion of actually treated patients (58/561) especially in single session group (29/487) may not be typical and represent the whole study population. Results showed that only one out of 25 potential variables (linear IAC length) was the significant predictor for hearing preservation after multisession GKRS, with a relatively high $\mathrm{P}$ value of $0.090(\mathrm{P}<0.1)$, which may be related to not enough cases and follow up periods of our present study. So, the preliminary results of present study need to be further validated with more cases in each group and long-term follow-up. More comparative studies with longer follow-up periods are also needed to address the optimal dose per session, number of fractions, and establish long-term safety and efficacy of this strategy. It's a pity that many patients lost in follow-up, thus we only included 29 patients in each group for this study. Finally, analysis regarded biological equivalent dose and accompanied adverse effects of accumulated shuttle dose of gamma knife with fractionation should be completed. Despite these uncertainties, multisession GKRS deserves to be considered as an effective strategy to increase the probability of hearing preservation for VS patients without reducing tumor control rate.

\section{Conclusions}

This study demonstrated that multisession GKRS is well tolerated and is an effective and acceptable treatment option for VS compared to single session radiosurgery. Hearing preservation rates after multisession GKRS were not statistically superior to single session GKRS in our shortterm follow-up study. The linear IAC length was the only prognostic factor for hearing preservation after GKRS in multisession group. Thus, patients with longer IAC length may benefit from a multisession strategy in terms of hearing preservation. However, prospective randomized controlled 
studies with long term follow-up are warranted to validate these findings.

\section{Acknowledgments}

We are grateful to Dr. Allen Ho from Stanford University for manuscript editing.

Funding: This work was supported by the grant from Health and Family Planning Commission of Shanghai Municipality (201640079).

\section{Footnote}

Conflicts of Interest: All authors have completed the ICMJE uniform disclosure form (available at http://dx.doi. org/10.21037/tcr.2018.08.15). The authors have no conflicts of interest to declare.

Ethical Statement: The authors are accountable for all aspects of the work in ensuring that questions related to the accuracy or integrity of any part of the work are appropriately investigated and resolved. The study was conducted in accordance with the Declaration of Helsinki (as revised in 2013). The study was conducted under the supervision of Ethics Committee of Huashan Hospital, Fudan University (IRB number: 20120302) and written informed consent was obtained from all patients.

Open Access Statement: This is an Open Access article distributed in accordance with the Creative Commons Attribution-NonCommercial-NoDerivs 4.0 International License (CC BY-NC-ND 4.0), which permits the noncommercial replication and distribution of the article with the strict proviso that no changes or edits are made and the original work is properly cited (including links to both the formal publication through the relevant DOI and the license). See: https://creativecommons.org/licenses/by-nc-nd/4.0/.

\section{References}

1. Hasegawa T, Kida Y, Kobayashi T, et al. Long-term outcomes in patients with vestibular schwannomas treated using gamma knife surgery: 10-year follow up. J Neurosurg 2005;102:10-6.

2. Lunsford LD, Niranjan A, Flickinger JC, et al. Radiosurgery of vestibular schwannomas: summary of experience in 829 cases. J Neurosurg 2005;102 Suppl:195-9.
3. Chopra R, Kondziolka D, Niranjan A, et al. Long-term follow-up of acoustic schwannoma radiosurgery with marginal tumor doses of 12 to 13 Gy. Int J Radiat Oncol Biol Phys 2007;68:845-51.

4. Miller RC, Foote RL, Coffey RJ, et al. Decrease in cranial nerve complications after radiosurgery for acoustic neuromas: a prospective study of dose and volume. Int J Radiat Oncol Biol Phys 1999;43:305-11.

5. Sun S, Liu A. Long-term follow-up studies of Gamma Knife surgery with a low margin dose for vestibular schwannoma. J Neurosurg 2012;117 Suppl:57-62.

6. van Eck AT, Horstmann GA. Increased preservation of functional hearing after gamma knife surgery for vestibular schwannoma. J Neurosurg 2005;102 Suppl:204-6.

7. Boari N, Bailo M, Gagliardi F, et al. Gamma Knife radiosurgery for vestibular schwannoma: clinical results at long-term follow-up in a series of 379 patients. J Neurosurg 2014;121 Suppl:123-42.

8. Han JH, Kim DG, Chung HT, et al. Hearing Outcomes After Stereotactic Radiosurgery for Vestibular Schwannomas: Mechanism of Hearing Loss and How to Preserve Hearing. Adv Tech Stand Neurosurg 2016;(43):3-36.

9. Iwai Y, Yamanaka K, Shiotani M, et al. Radiosurgery for acoustic neuromas: results of low-dose treatment. Neurosurgery 2003;53:282-87.

10. Kano H, Kondziolka D, Khan A, et al. Predictors of hearing preservation after stereotactic radiosurgery for acoustic neuroma. J Neurosurg 2009;111:863-73.

11. Kim KM, Park CK, Chung HT, et al. Long-term Outcomes of Gamma Knife Stereotactic Radiosurgery of Vestibular Schwannomas. J Korean Neurosurg Soc 2007;42:286-92.

12. Paek SH, Chung HT, Jeong SS, et al. Hearing preservation after gamma knife stereotactic radiosurgery of vestibular schwannoma. Cancer 2005;104:580-90.

13. Pollock BE, Driscoll CL, Foote RL, et al. Patient outcomes after vestibular schwannoma management: a prospective comparison of microsurgical resection and stereotactic radiosurgery. Neurosurgery 2006;59:77-85.

14. Yang I, Sughrue ME, Han SJ, et al. A comprehensive analysis of hearing preservation after radiosurgery for vestibular schwannoma: clinical article. J Neurosurg 2013;119 Suppl:851-9.

15. Brown M, Ruckenstein M, Bigelow D, et al. Predictors of hearing loss after gamma knife radiosurgery for vestibular schwannomas: age, cochlear dose, and tumor coverage. Neurosurgery 2011;69:605-13.

16. Linskey ME, Johnstone PA, O'Leary M, et al. Radiation 
exposure of normal temporal bone structures during stereotactically guided gamma knife surgery for vestibular schwannomas. J Neurosurg 2003;98:800-6.

17. Massager N, Nissim O, Delbrouck C, et al. Irradiation of cochlear structures during vestibular schwannoma radiosurgery and associated hearing outcome. J Neurosurg 2007;107:733-9.

18. Massager N, Nissim O, Delbrouck C, et al. Role of intracanalicular volumetric and dosimetric parameters on hearing preservation after vestibular schwannoma radiosurgery. Int J Radiat Oncol Biol Phys 2006;64:1331-40.

19. Timmer FC, Hanssens PE, van Haren AE, et al. Gamma knife radiosurgery for vestibular schwannomas: results of hearing preservation in relation to the cochlear radiation dose. Laryngoscope 2009;119:1076-81.

20. Akpinar B, Mousavi SH, McDowell MM, et al. Early Radiosurgery Improves Hearing Preservation in Vestibular Schwannoma Patients With Normal Hearing at the Time of Diagnosis. Int J Radiat Oncol Biol Phys 2016;95:729-34.
21. Adler JR, Jr, Gibbs IC, Puataweepong P, et al. Visual field preservation after multisession cyberknife radiosurgery for perioptic lesions. Neurosurgery 2006;59:244-54.

22. Kim JW, Im YS, Nam DH, et al. Preliminary report of multisession gamma knife radiosurgery for benign perioptic lesions: visual outcome in 22 patients. J Korean Neurosurg Soc 2008;44:67-71.

23. Gardner G, Robertson JH. Hearing preservation in unilateral acoustic neuroma surgery. Ann Otol Rhinol Laryngol 1988;97:55-66.

24. Yang I, Sughrue ME, Han SJ, et al. A comprehensive analysis of hearing preservation after radiosurgery for vestibular schwannoma. J Neurosurg 2010;112:851-9.

25. Linskey ME. Hearing preservation in vestibular schwannoma stereotactic radiosurgery: what really matters? J Neurosurg 2008;109 Suppl:129-36.

26. Rashid A, Karam SD, Rashid B, et al. Multisession Radiosurgery for Hearing Preservation. Semin Radiat Oncol 2016;26:105-11.
Cite this article as: Tang $\mathrm{X}$, Zheng $M$, Tang H, Wu H, Zhang N, Dai J, Pan L. Hearing outcomes between multi-session and single-session radiosurgery for vestibular schwannoma: a single center study. Transl Cancer Res 2018;7(4):1092-1102. doi: 10.21037/tcr.2018.08.15 